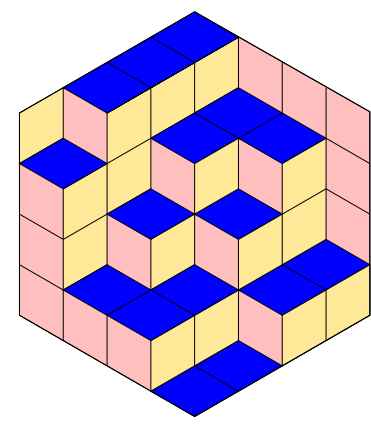

Arindam Biswas \& Jyoti Prakash Saha

A Cheeger type inequality in finite Cayley sum graphs

Volume 4, issue 3 (2021), p. 517-531.

<http://alco.centre-mersenne.org/item/ALCO_2021__4_3_517_0>

(C) The journal and the authors, 2021.

Some rights reserved.

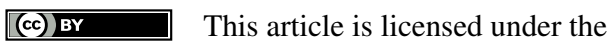

Creative Commons ATtRibution 4.0 InTERnATIONAL LiCEnSE.

http://creativecommons.org/licenses/by/4.0/

Access to articles published by the journal Algebraic Combinatorics on the website http://alco.centre-mersenne.org/ implies agreement with the Terms of Use (http://alco.centre-mersenne.org/legal/).

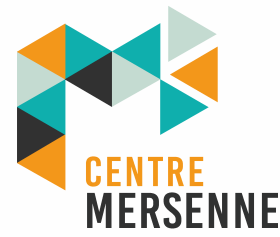

Algebraic Combinatorics is member of the Centre Mersenne for Open Scientific Publishing www.centre-mersenne.org 


\title{
A Cheeger type inequality in finite Cayley sum graphs
}

\author{
Arindam Biswas \& Jyoti Prakash Saha
}

\begin{abstract}
Let $G$ be a finite group with $|G| \geqslant 4$ and $S$ be a subset of $G$ with $|S|=d$ such that the Cayley sum graph $C_{\Sigma}(G, S)$ is undirected and connected. We show that the nontrivial spectrum of the normalised adjacency operator of $C_{\Sigma}(G, S)$ is controlled by its Cheeger constant and its degree. We establish an explicit lower bound for the non-trivial spectrum of these graphs, namely, the non-trivial eigenvalues of the normalised adjacency operator lies in the interval $\left(-1+\frac{h_{\Sigma}(G)^{4}}{\eta}, 1-\frac{h_{\Sigma}(G)^{2}}{2 d^{2}}\right]$, where $h_{\Sigma}(G)$ denotes the vertex Cheeger constant of the $d$-regular graph $C_{\Sigma}(G, S)$ and $\eta=2^{9} d^{8}$. Further, we improve upon a recently obtained bound on the non-trivial spectrum of the normalised adjacency operator of the Cayley graph of finite groups.
\end{abstract}

\section{INTRODUCTION}

Let $G$ be a finite group, and $S$ be a subset of $G$ with $|S|=d$. The Cayley sum graph $C_{\Sigma}(G, S)$ is the graph having $G$ as its set of vertices and for $g, h \in G$, the vertex $h$ is adjacent to $g$ if $h=g^{-1} s$ for some element $s \in S$. These are classical combinatorial objects. We also recall that the Cayley graph of $G$ (sometimes, called the Cayley difference graph), denoted by $C(G, S)$, is the graph having $G$ as its set of vertices and a vertex $h$ is adjacent to a vertex $g$ if $h=g s$ for some element $s \in S$. The structures of $C(G, S)$ and $C_{\Sigma}(G, S)$ can be very different. This can be seen by considering the Cayley graph $C(G, S)$ and the Cayley sum graph $C_{\Sigma}(G, S)$ of $G=\mathbb{Z} / n \mathbb{Z}(n \geqslant 5)$ with respect to the set $S=\{ \pm 1\}$. The former is always a cycle graph while the latter need not be so (for instance, the latter is a cycle graph when $n$ is even and is a path with loops at the endpoints whenever $n$ is odd). Cayley graphs have been extensively studied over the ages. However, despite being classical combinatorial objects, the literature on Cayley sum graphs is not extensive. Very few things are known about Cayley sum graphs and most of them are quite recent works. They include those of Chung [7], Green [11, 12], Green-Morris [13], Grynkiewicz-Lev-Serra [14], DeVos-Goddyn-Mohar-Šámal [9], Mrazović [18], Konyagin-Shkredov [15], Ma-FengWang [17]. Indeed, even the question of vertex connectivity of abelian Cayley sum graphs was treated very recently in 2009 by Grynkiewicz-Lev-Serra [14], whereas vertex connectivity of abelian Cayley graphs is relatively easy. One reason for this fact is that unlike in the case of Cayley graphs, Cayley sum graphs may have less symmetry in them. Even less is known about the spectra of Cayley sum graphs. In fact, among the previous works, only that of Chung [7] and that of DeVos et. al. [9]

Manuscript received 5th June 2020, revised and accepted 20th December 2020.

KEYwords. Expander graphs, Cheeger inequality, Spectra of Cayley sum graphs. 
deal with eigenvalues of some Cayley sum graphs, whereas the computation of distribution of eigenvalues of graphs is a fundamental topic of interest in graph theory. It is not yet known whether random Cayley sum graphs are expanders. Much remains to be discovered about Cayley sum graphs and it is a topic of current research. In this article, our main motivation is to establish a lower bound on the distribution of non-trivial eigenvalues of Cayley sum graphs.

In the following, the graphs and the multi-graphs considered are all undirected. The multi-graphs may possibly admit multiple edges. Moreover, the graphs and the multigraphs considered may admit loops. Given a finite $d$-regular multi-graph $\mathbb{G}=(V, E)$ where $V$ denotes the set of vertices and $E \subseteq\left(\begin{array}{c}V \\ 2\end{array}\right)$ denotes the multi-set of edges, we have the normalised adjacency matrix $T$ of size $|V| \times|V|$, which is equal to $\frac{1}{d} A$, where $A$ denote the adjacency matrix of $\mathbb{G}$ of size $|V| \times|V|$, whose $(i, j)$-th entry is equal to the number of edges joining the $i$-th vertex and the $j$-th vertex of $V$. Note that the eigenvalues of $T$ lie in the interval $[-1,1]$. The normalised Laplacian matrix of $\mathbb{G}$ is defined by

$$
L:=I_{|V|}-T
$$

where $I_{|V|}$ denotes the identity matrix of size $|V| \times|V|$. The eigenvalues of $L$ lie in the interval $[0,2]$. Denote the eigenvalues of $T$ and the eigenvalues of $L$ by $\left\{t_{i}: i=\right.$ $1, \ldots,|V|\}$ and $\left\{\lambda_{i}: i=1, \ldots,|V|\right\}$ respectively, such that $\lambda_{i}=1-t_{i}$ and

$$
\begin{aligned}
& -1 \leqslant t_{n} \leqslant t_{n-1} \leqslant \cdots \leqslant t_{2} \leqslant t_{1}=1, \\
& 0=\lambda_{1} \leqslant \lambda_{2} \leqslant \cdots \leqslant \lambda_{n-1} \leqslant \lambda_{n} \leqslant 2 .
\end{aligned}
$$

The multi-graph $\mathbb{G}$ is connected if and only if $\lambda_{2}>0$ (equivalently, $t_{2}<1$ ). Moreover, if $\mathbb{G}$ is connected, then it is bipartite if and only if $\lambda_{n}=2$ (equivalently, $t_{n}=-1$ ).

For a subset $V_{1} \subseteq V$, we denote the neighbourhood of $V_{1}$ by $N\left(V_{1}\right)$ where,

$$
N\left(V_{1}\right):=\left\{v \in V:\left\{v, v_{1}\right\} \in E \text { for some } v_{1} \in V_{1}\right\}
$$

The boundary of $V_{1}$ is defined as $\delta\left(V_{1}\right):=N\left(V_{1}\right) \backslash V_{1}$.

REMARK 1.1. In the case of a Cayley graph $C(G, S)$, the neighbourhood $N\left(V_{1}\right)$ of a subset $V_{1}$ of $G$ is equal to $V_{1} S$ and its boundary $\delta\left(V_{1}\right)$ is equal to $V_{1} S \backslash S$, while for a Cayley sum graph $C_{\Sigma}(G, S)$, the neighbourhood $N\left(V_{1}\right)$ of a subset $V_{1}$ of $G$ is equal to $V_{1}^{-1} S$, and its boundary $\delta\left(V_{1}\right)$ is equal to $V_{1}^{-1} S \backslash S$.

Definition 1.2 (Vertex Cheeger constant). The vertex Cheeger constant of the multigraph $\mathbb{G}=(V, E)$ with $|V| \geqslant 2$, denoted by $h(\mathbb{G})$, is defined as

$$
h(\mathbb{G}):=\inf \left\{\frac{\left|\delta\left(V_{1}\right)\right|}{\left|V_{1}\right|}: \varnothing \neq V_{1} \subseteq V,\left|V_{1}\right| \leqslant \frac{|V|}{2}\right\} .
$$

By considering a subset of $V$ having size around half of the size of $V$, it follows that the vertex Cheeger constant of $(V, E)$ cannot be much bigger than one. Indeed, if $V$ contains an even number of elements, then taking a subset $V_{1}$ of $V$ of size equal to $|V| / 2$, one observes that the ratio of the size of its boundary $\delta\left(V_{1}\right)$ to the size of $V_{1}$ is bounded from the above by 1 . Moreover, if $V$ contains an odd number of elements, then taking a subset $V_{2}$ of $V$ of size equal to $(|V|-1) / 2$, one observes that the ratio of the size of its boundary $\delta\left(V_{2}\right)$ to its size $\left|V_{2}\right|$ is bounded from the above by

$$
\frac{\left|V \backslash V_{2}\right|}{\left|V_{2}\right|}=\frac{|V|+1}{|V|-1}=1+\frac{2}{|V|-1} .
$$

In particular, the vertex Cheeger constant of $\mathbb{G}$ satisfies $h(\mathbb{G}) \leqslant 2$.

Next, we recall the notion of an expander graph as stated by Alon in [1]. 
Definition $1.3((n, d, \varepsilon)$-expander $)$. Let $\varepsilon>0$. An $(n, d, \varepsilon)$-expander is a graph $\mathbb{G}=$ $(V, E)$ on $|V|=n$ vertices, having maximal degree $d$, such that for every set $\varnothing \neq V_{1} \subseteq$ $V$ satisfying $\left|V_{1}\right| \leqslant \frac{n}{2},\left|\delta\left(V_{1}\right)\right| \geqslant \varepsilon\left|V_{1}\right|$ holds (equivalently, $h(\mathbb{G}) \geqslant \varepsilon$ ).

It was shown qualitatively by Breuillard, Green, Guralnick, and Tao that the eigenvalues of the normalised Laplacian matrix of non-bipartite finite Cayley graphs are bounded away from 2 [4, Appendix E]. Based on their arguments, the first author recently established an explicit upper bound [3, Theorem 1.4]. In this article, we show that a similar phenomenon occurs for the spectrum of the Cayley sum graph $C_{\Sigma}(G, S)$ by suitably adapting the strategy outlined in [4, Appendix E], along with the introduction of some new refinements. For an outline of the proof, we refer to $\S 1.1$. Henceforth, we assume that $|G| \geqslant 4$, to avoid trivial cases.

THEOREM 1.4. Let $h_{\Sigma}(G)$ denote the vertex Cheeger constant of the connected Cayley sum graph $C_{\Sigma}(G, S)$. Then if $C_{\Sigma}(G, S)$ is non-bipartite, we have

$$
\left.\lambda_{n}<2-\frac{h_{\Sigma}(G)^{4}}{2^{9} d^{8}} \text { (equivalently, }-1+\frac{h_{\Sigma}(G)^{4}}{2^{9} d^{8}}<t_{n}\right),
$$

where $\lambda_{n}$ (respectively, $t_{n}$ ) is the largest (respectively, the smallest) eigenvalue of the normalised Laplacian matrix (respectively, the normalised adjacency matrix) of $C_{\Sigma}(G, S)$.

REMARK 1.5. Note that when $C_{\Sigma}(G, S)$ is bipartite, the spectrum of its adjacency matrix is symmetric about the origin. In this case, the lower spectrum is determined by the upper spectrum, for instance, $t_{n}=-t_{1}=-1$ and $t_{n-1}=-t_{2} \geqslant-1+\frac{h_{\Sigma}(G)^{2}}{2 d^{2}}$, which follows from the discrete Cheeger-Buser inequality. Theorem 1.4 focuses on the non-bipartite Cayley sum graphs and shows that the smallest eigenvalue of the normalised adjacency matrix admits a lower bound depending only on the vertex Cheeger constant and the degree. This result is deduced after the proof of Theorem 2.10.

One has the following corollary of Theorem 1.4.

Corollary 1.6. Let $d \geqslant 2$ be an integer. Let $\left\{C_{\Sigma}\left(G_{k}, S_{k}\right)\right\}_{k \geqslant 1}$ be a sequence of nonbipartite, finite Cayley sum graphs with $\left|G_{k}\right| \rightarrow \infty,\left|S_{k}\right|=d$. Then, if there exists an uniform $\varepsilon>0$, such that each graph $C_{\Sigma}\left(G_{k}, S_{k}\right)$ in the sequence is an $\left(\left|G_{k}\right|, d, \varepsilon\right)$ expander, we have all the eigenvalues of the normalised adjacency matrix of each graph are uniformly bounded away from -1 .

As a by-product of our proof, we improve the bound established for Cayley graphs in [3, Theorem 1.4]. See Theorem 2.12.

1.1. Outline OF The PROOF. We outline the proof of Theorem 1.4. To prove this result, we assume on the contrary that the normalised adjacency matrix $T$ of the Cayley sum graph admits an eigenvalue close to -1 (see Theorem 2.10). This implies that $T^{2}$ has an eigenvalue close to 1 . We define a multi-graph $\mathcal{M}$ such that its normalised adjacency matrix is equal to $T^{2}$ (see the proof of Proposition 2.8). Then the discrete Cheeger-Buser inequality yields an upper bound on the edge-Cheeger constant of $\mathcal{M}$, which in turn implies an upper bound on the vertex Cheeger constant of $\mathcal{M}$. This yields a subset $A$ of $G$ of size $\leqslant \frac{|G|}{2}$ having a convenient upper bound on $\left|S^{-1} A S \backslash A\right| /|A|$. Using combinatorial arguments, we obtain upper bounds on the sizes of several subsets defined using $A$ (see Proposition 2.8). As a consequence, for a given element $g \in G$, we establish a dichotomy result on the size of $A \cap A g$ (see Proposition 2.9), which states that the size $A \cap A g$ is either very small or quite large as compared to the size of $A$. This allows us to adapt an argument due to Freĭman [10] in our set-up to construct a subgroup $H_{+}$of $G$ (see Theorem 2.10). From the bound on 
the smallest eigenvalue of $T$, it follows that the subgroup $H_{+}$has index two in $G$. In Proposition 2.9, we also establish a similar dichotomy result on the size of $A \cap A^{-1} g$. Using the strategy of Fre mman once again, we define a subset $H_{-}$of $G$, which avoids $S$ and is equal to a coset of $H_{+}$in $G$, i.e. to $H_{+}$or $G \backslash H_{+}$. To conclude the result, we consider two cases. First, if $H_{-}$is equal to $H_{+}$, then the index two subgroup $H_{+}$avoids $S$, which contradicts the hypothesis that $C_{\Sigma}(G, S)$ is non-bipartite (by Lemma 2.5). Next, if $H_{-}$is equal to $G \backslash H_{+}$, then the index two subgroup $H_{+}$contains $S$, which contradicts the hypothesis that $C_{\Sigma}(G, S)$ is connected.

\section{Proof of the MAIN RESUlT}

The degree of a vertex of a multi-graph is the number of half-edges adjacent to it (in the absence of loops). The presence of a loop at a vertex increases its degree by one. A multi-graph is said to be $r$-regular if each vertex has degree $r$. Apart from the vertex expansion as in Definition 1.3, we also have the notion of edge expansion.

Definition 2.1 (Edge expansion). Let $\mathbb{G}=(V, E)$ be a d-regular multi-graph with vertex set $V$ and edge multi-set $E$. For a subset $\varnothing \neq V_{1} \subseteq V$, let $E\left(V_{1}, V \backslash V_{1}\right)$ be the edge boundary of $V_{1}$, defined as

$$
E\left(V_{1}, V \backslash V_{1}\right):=\left\{\left(v_{1}, v_{2}\right) \in E: v_{1} \in V, v_{2} \in V \backslash V_{1}\right\}
$$

Then the edge expansion ratio $\phi\left(V_{1}\right)$ of $V_{1}$ is defined as

$$
\phi\left(V_{1}\right):=\frac{\left|E\left(V_{1}, V \backslash V_{1}\right)\right|}{d\left|V_{1}\right|} .
$$

Definition 2.2 (Edge-Cheeger constant). For a multi-graph $\mathbb{G}=(V, E)$, its edgeCheeger constant $\mathfrak{h}(\mathbb{G})$ is defined by

$$
\mathfrak{h}(\mathbb{G}):=\inf _{\varnothing \neq V_{1} \subseteq V,\left|V_{1}\right| \leqslant|V| / 2} \phi\left(V_{1}\right) .
$$

In a $d$-regular multi-graph, the two Cheeger constants are related by the following.

LEMma 2.3. Let $\mathbb{G}=(V, E)$ be a d-regular multi-graph. Then

$$
\frac{h(\mathbb{G})}{d} \leqslant \mathfrak{h}(\mathbb{G}) \leqslant h(\mathbb{G})
$$

holds.

Proof. Let $\varnothing \neq V_{1} \subseteq V$ and we consider the map

$$
\psi: E\left(V_{1}, V \backslash V_{1}\right) \rightarrow \delta\left(V_{1}\right) \text { given by }\left(v_{1}, v_{2}\right) \mapsto v_{2} .
$$

This map is surjective, hence we have the left hand side and at the worst case $d$ to 1 wherein we get the right hand side.

The discrete Cheeger-Buser inequality relates the (edge) Cheeger constant with the second smallest eigenvalue of the Laplacian matrix. It is the version for graphs of the corresponding inequalities for the Laplace-Beltrami operator on compact Riemannian manifolds. It was first proven by Cheeger [6] (the lower bound) and by Buser [5] (the upper bound). The discrete version was shown by Alon and Milman [2] (Proposition 2.4). 
Proposition 2.4 (Discrete Cheeger-Buser inequality). Let $\mathbb{G}=(V, E)$ be a finite d-regular multi-graph. Let $\lambda_{2}$ denote the second smallest eigenvalue of its normalised Laplacian matrix and $\mathfrak{h}(\mathbb{G})$ be the edge-Cheeger constant. Then

$$
\frac{\mathfrak{h}(\mathbb{G})^{2}}{2} \leqslant \lambda_{2} \leqslant 2 \mathfrak{h}(\mathbb{G})
$$

Proof. See [16, Proposition 4.2.4, 4.2.5] or [8, Section 3].

LEMMA 2.5. If $G$ contains a subgroup of index two which does not intersect $S$, then the Cayley sum graph $C_{\Sigma}(G, S)$ is bipartite.

Proof. Suppose $G$ contains a subgroup $H$ of index two which does not intersect $S$. Then $H^{-1} S$ is equal to $G \backslash H$ and hence $H$ is an independent set. Moreover, $(G \backslash$ $H)^{-1} S$ is equal to $H$. Thus, $G \backslash H$ is also an independent set. So, the Cayley sum graph $C_{\Sigma}(G, S)$ is bipartite.

Lemma 2.6. The Cayley sum graph $C_{\Sigma}(G, S)$ is undirected if and only if $S$ is closed under conjugation.

Proof. The graph $C_{\Sigma}(G, S)$ is undirected if and only if for any $g \in G$ and $s \in S$, $\left(g^{-1} s\right)^{-1} t=g$ holds for some $t \in S$, which is equivalent to $S$ being closed under conjugation.

Proposition 2.7. Suppose $(V, E)$ is an $(n, d, \varepsilon)$-expander with $\varepsilon>0$ and $|V| \geqslant 4$. Let $A$ be a subset of $V$ with $|A| \geqslant \frac{1}{2}|V|$. Then, the inequalities

$$
\begin{gathered}
\varepsilon \leqslant d-1, \\
|\delta(A)| \geqslant \frac{\varepsilon}{d}|V \backslash A|
\end{gathered}
$$

hold.

Proof. If $u, v$ are two adjacent vertices in $V$, then it follows that $\varepsilon|\{u, v\}| \leqslant$ $|\delta(\{u, v\})| \leqslant 2(d-1)$ (since the size of $\{u, v\}$ is $\leqslant \frac{1}{2}|V|$ ), which shows that $\varepsilon \leqslant d-1$.

Let $B$ denote the subset $V \backslash(A \cup \delta(A))$ of $V$. Suppose $B$ is nonempty. Since $|A| \geqslant \frac{1}{2}|V|$, it follows that $|B| \leqslant \frac{1}{2}|V|$. This shows that $\varepsilon|B| \leqslant|\delta(B)|$. Note that $V$ is equal to the union of the pairwise disjoint subsets $A, \delta(A), B$. Since no element of $A$ is adjacent to an element of $B$, it follows that $\delta(B)$ is contained in $\delta(A)$. This shows that $\varepsilon|B| \leqslant|\delta(B)| \leqslant|\delta(A)|$. Suppose (2) does not hold, i.e. the inequality $d|\delta(A)|<\varepsilon(|B|+|\delta(A)|)$ holds. This yields

$$
(d-\varepsilon) \varepsilon|B| \leqslant(d-\varepsilon)|\delta(A)|<\varepsilon|B|,
$$

which implies that $\varepsilon>d-1$, which is impossible. Hence, the set $B$ is empty. Then (2) follows from the inequality $\varepsilon \leqslant d$, which holds by (1).

Proposition 2.8. Let $C_{\Sigma}(G, S)$ be a non-bipartite $(n, d, \varepsilon)$-vertex expander for some $\varepsilon>0$. Suppose the normalised adjacency matrix of $C_{\Sigma}(G, S)$ has an eigenvalue in the interval $(-1,-1+\zeta]$ for some $\zeta$ satisfying $0<\zeta \leqslant \frac{\varepsilon^{2}}{4 d^{4}}$. Then for some subset $A$ of $G$, the following conditions hold with $\beta=d^{2} \sqrt{2 \zeta(2-\zeta)}$.

(1) $\frac{1}{2+\beta+\frac{d \beta}{\varepsilon}}|G| \leqslant|A| \leqslant \frac{1}{2}|G|$.

(2) $\left|A g \cap(A g)^{-1} S\right| \leqslant \frac{\beta}{\varepsilon}|A|$ for all $g \in G$.

(3) $\left|(A g)^{-1} s \Delta(A g)^{c}\right| \leqslant \frac{\beta}{\varepsilon}(\varepsilon+d+2)|A|$ for all $s \in S, g \in G$.

(4) $\left|A^{-1} g \cap\left(A^{-1} g\right)^{-1} S\right| \leqslant \frac{\beta}{\varepsilon}|A|$ for all $g \in G$.

(5) $\left|\left(A^{-1} g\right)^{-1} s \Delta\left(A^{-1} g\right)^{c}\right| \leqslant \frac{\beta}{\varepsilon}(\varepsilon+d+2)|A|$ for all $s \in S, g \in G$. 
Proof. Since $|G| \geqslant 4$, it follows from Proposition 2.7 that

$$
\varepsilon \leqslant d-1
$$

This implies that $\zeta<1$. Let $T$ denote the normalised adjacency matrix of the Cayley sum graph $C_{\Sigma}(G, S)$. Since $T$ has an eigenvalue in $(-1,-1+\zeta]$ and $\zeta<1$, it follows that $T^{2}$ has an eigenvalue $\nu$ in $\left[(1-\zeta)^{2}, 1\right)$. Let $\mathcal{M}$ denote the weighted graph having $G$ as its set of vertices and having $T^{2}$ as its normalised adjacency matrix. Thus the second largest eigenvalue of the normalised adjacency matrix of $\mathcal{M}$ is $\geqslant \nu \geqslant(1-\zeta)^{2}=$ $1-\zeta(2-\zeta)$. Hence the second smallest eigenvalue of the normalised Laplacian matrix of $\mathcal{M}$ is $\leqslant \zeta(2-\zeta)$. By the discrete Cheeger-Buser inequality (Proposition 2.4), it follows that the edge-Cheeger constant of $\mathcal{M}$ satisfies

$$
\frac{1}{2} \mathfrak{h}(\mathcal{M})^{2} \leqslant \zeta(2-\zeta)
$$

which yields

$$
\mathfrak{h}(\mathcal{M}) \leqslant \sqrt{2 \zeta(2-\zeta)}
$$

Consequently, by Lemma 2.3, the vertex Cheeger constant of $\mathcal{M}$ satisfies

$$
h(\mathcal{M}) \leqslant d^{2} \mathfrak{h}(\mathcal{M}) \leqslant d^{2} \sqrt{2 \zeta(2-\zeta)}
$$

This implies that for some non-empty subset $A$ of $G$ with $|A| \leqslant \frac{1}{2}|G|$,

$$
\frac{\left|S^{-1} A S \backslash A\right|}{|A|} \leqslant d^{2} \sqrt{2 \zeta(2-\zeta)}
$$

holds.

We claim that

$$
\left|A \cup A^{-1} S\right| \geqslant \frac{1}{2}|G|
$$

Otherwise, the inequality $\left|A \cup A^{-1} S\right| \leqslant \frac{1}{2}|G|$ would imply

$$
\varepsilon\left|A \cup A^{-1} S\right| \leqslant\left|\left(\left(A \cup A^{-1} S\right)^{-1} S\right) \backslash\left(A \cup A^{-1} S\right)\right|,
$$

which combined with the inequalities

$$
\varepsilon|A| \leqslant \varepsilon\left|A \cup A^{-1} S\right|
$$

and

$$
\begin{aligned}
\left|\left(\left(A \cup A^{-1} S\right)^{-1} S\right) \backslash\left(A \cup A^{-1} S\right)\right| & =\left|\left(A^{-1} S \cup S^{-1} A S\right) \backslash\left(A \cup A^{-1} S\right)\right| \\
& \leqslant\left|S^{-1} A S \backslash A\right| \\
& \leqslant|A| d^{2} \sqrt{2 \zeta(2-\zeta)}
\end{aligned}
$$

implies

$$
\varepsilon \leqslant d^{2} \sqrt{2 \zeta(2-\zeta)}<d^{2} \sqrt{4 \zeta}
$$

This contradicts the assumption $\zeta \leqslant \frac{\varepsilon^{2}}{4 d^{4}}$. Hence $(5)$ holds.

Applying Proposition 2.7 to the Cayley sum graph $C_{\Sigma}(G, S)$, we obtain $\frac{\varepsilon}{d}\left|G \backslash\left(A \cup A^{-1} S\right)\right| \leqslant\left|\left(\left(A \cup A^{-1} S\right)^{-1} S\right) \backslash\left(A \cup A^{-1} S\right)\right| \leqslant|A| d^{2} \sqrt{2 \zeta(2-\zeta)}=|A| \beta$. 
So

$$
\frac{d \beta}{\varepsilon}|A| \geqslant\left|G \backslash\left(A \cup A^{-1} S\right)\right|=|G|-\left|A \cup A^{-1} S\right|,
$$

which implies

$$
\begin{aligned}
|G| & \leqslant \frac{d \beta}{\varepsilon}|A|+\left|A \cup A^{-1} S\right| \\
& \leqslant \frac{d \beta}{\varepsilon}|A|+|A|+\left|A^{-1} S\right| \\
& =\frac{d \beta}{\varepsilon}|A|+|A|+\left|S^{-1} A\right| \\
& \leqslant \frac{d \beta}{\varepsilon}|A|+|A|+\left|S^{-1} A S\right| \\
& \leqslant \frac{d \beta}{\varepsilon}|A|+|A|+|A|+\left|S^{-1} A S \backslash A\right| \\
& \leqslant \frac{d \beta}{\varepsilon}|A|+2|A|+\beta|A|,
\end{aligned}
$$

where the last inequality follows from (4). This proves the inequalities as in statement (1).

To obtain the inequality in statement (2), note that $|A| \leqslant \frac{1}{2}|G|$ implies that $\mid A g \cap$ $(A g)^{-1} S\left|\leqslant \frac{1}{2}\right| G \mid$. Since $C_{\Sigma}(G, S)$ is an $\varepsilon$-vertex expander, it follows that

$$
\begin{aligned}
\varepsilon\left|A g \cap(A g)^{-1} S\right| & \leqslant\left|\left(\left(A g \cap(A g)^{-1} S\right)^{-1} S\right) \backslash\left(A g \cap(A g)^{-1} S\right)\right| \\
& =\left|\left(\left((A g)^{-1} \cap S^{-1} A g\right) S\right) \backslash\left(A g \cap(A g)^{-1} S\right)\right| \\
& \leqslant\left|\left((A g)^{-1} S \cap S^{-1} A g S\right) \backslash\left(A g \cap(A g)^{-1} S\right)\right| \\
& \leqslant\left|S^{-1} A g S \backslash A g\right| \\
& =\left|S^{-1} A g S g^{-1} \backslash A\right| \\
& =\left|S^{-1} A S \backslash A\right| \\
& \leqslant \beta|A| .
\end{aligned}
$$

This establishes the inequality in statement (2).

To obtain the inequality in statement (3), it suffices to observe that

$$
\begin{aligned}
\left|(A g)^{-1} s \Delta(A g)^{c}\right| & =\left|(A g)^{-1} s\right|+\left|(A g)^{c}\right|-2\left|(A g)^{-1} s \cap(A g)^{c}\right| \\
& =|A g|+|G|-|A g|-2\left(\left|(A g)^{-1} s\right|-\left|(A g)^{-1} s \cap A g\right|\right) \\
& =|G|-2\left|(A g)^{-1} s\right|+2\left|(A g)^{-1} s \cap A g\right| \\
& =|G|-2|A|+2\left|A g \cap(A g)^{-1} s\right| \\
& \leqslant|G|-2|A|+2\left|A g \cap(A g)^{-1} S\right| \\
& \leqslant\left(2+\beta+\frac{d \beta}{\varepsilon}\right)|A|-2|A|+\frac{2 \beta}{\varepsilon}|A| \\
& =\beta\left(1+\frac{d}{\varepsilon}+\frac{2}{\varepsilon}\right)|A|
\end{aligned}
$$

holds, where the final inequality is obtained by applying statements (1) and (2). 
To obtain the inequality in statement (4), note that $\left|A^{-1}\right| \leqslant \frac{1}{2}|G|$ implies that $\left|A^{-1} g \cap\left(A^{-1} g\right)^{-1} S\right| \leqslant \frac{1}{2}|G|$. Since $C_{\Sigma}(G, S)$ is an $\varepsilon$-vertex expander, it follows that

$$
\begin{aligned}
\varepsilon\left|A^{-1} g \cap\left(\left(A^{-1} g\right)^{-1} S\right)\right| & \leqslant\left|\left(\left(A^{-1} g \cap\left(\left(A^{-1} g\right)^{-1} S\right)\right)^{-1} S\right) \backslash\left(A^{-1} g \cap\left(\left(A^{-1} g\right)^{-1} S\right)\right)\right| \\
& =\mid\left(\left(\left(A^{-1} g\right)^{-1} S \cap\left(S^{-1} A^{-1} g S\right)\right) \backslash\left(A^{-1} g \cap\left(\left(A^{-1} g\right)^{-1} S\right)\right) \mid\right. \\
& \leqslant\left|\left(\left(\left(A^{-1} g\right)^{-1} S\right) \cap\left(S^{-1} A^{-1} g S\right)\right) \backslash\left(A^{-1} g \cap\left(\left(A^{-1} g\right)^{-1} S\right)\right)\right| \\
& \leqslant\left|S^{-1} A^{-1} g S \backslash A^{-1} g\right| \\
& =\left|S^{-1} A^{-1} g S g^{-1} \backslash A^{-1}\right| \\
& =\left|S^{-1} A^{-1} S \backslash A^{-1}\right| \\
& =\left|S^{-1} A S \backslash A\right| \\
& \leqslant \beta|A| .
\end{aligned}
$$

This establishes the inequality in statement (4).

To complete the proof, it suffices to observe that

$$
\begin{aligned}
\left|\left(A^{-1} g\right)^{-1} s \Delta\left(A^{-1} g\right)^{c}\right| & =\left|\left(A^{-1} g\right)^{-1} s\right|+\left|\left(A^{-1} g\right)^{c}\right|-2\left|\left(A^{-1} g\right)^{-1} s \cap\left(A^{-1} g\right)^{c}\right| \\
& =\left|A^{-1} g\right|+|G|-\left|A^{-1} g\right|-2\left(\left|\left(A^{-1} g\right)^{-1} s\right|-\left|\left(A^{-1} g\right)^{-1} s \cap A^{-1} g\right|\right) \\
& =|G|-2\left|\left(A^{-1} g\right)^{-1} s\right|+2\left|\left(A^{-1} g\right)^{-1} s \cap A^{-1} g\right| \\
& =|G|-2|A|+2\left|A^{-1} g \cap\left(A^{-1} g\right)^{-1} s\right| \\
& \leqslant|G|-2|A|+2\left|A^{-1} g \cap\left(\left(A^{-1} g\right)^{-1} S\right)\right| \\
& \leqslant\left(2+\beta+\frac{d \beta}{\varepsilon}\right)|A|-2|A|+\frac{2 \beta}{\varepsilon}|A| \\
& =\beta\left(1+\frac{d}{\varepsilon}+\frac{2}{\varepsilon}\right)|A|
\end{aligned}
$$

holds, where the final inequality is obtained by applying statement (1) and (4).

Proposition 2.9. Under the notations and assumptions as in Proposition 2.8, and the additional hypothesis

$$
\beta<\frac{\varepsilon^{2}}{4 d(d+1)}
$$

it follows that for a given element $g \in G$,

(1) exactly one of the inequalities

$$
|A \cap A g| \leqslant \frac{d \beta}{\varepsilon^{2}}(\varepsilon+d+2)|A|, \quad|A \cap A g| \geqslant\left(1-\frac{d \beta}{\varepsilon^{2}}(\varepsilon+d+2)\right)|A|
$$

holds, and

(2) exactly one of the inequalities

$$
\left|A \cap A^{-1} g\right| \leqslant \frac{d \beta}{\varepsilon^{2}}(\varepsilon+d+2)|A|, \quad\left|A \cap A^{-1} g\right| \geqslant\left(1-\frac{d \beta}{\varepsilon^{2}}(\varepsilon+d+2)\right)|A|
$$

holds.

Proof. Note that the inequalities

$$
\frac{2 d \beta}{\varepsilon^{2}}(\varepsilon+d+2) \leqslant \frac{2 d \beta}{\varepsilon^{2}}(d+d+2)=\frac{4 d \beta}{\varepsilon^{2}}(d+1)<1
$$

imply that

$$
\frac{d \beta}{\varepsilon^{2}}(\varepsilon+d+2)<1-\frac{d \beta}{\varepsilon^{2}}(\varepsilon+d+2) .
$$


Hence it suffices to show that for a given element $g \in G$, one of the inequalities

$$
|A \cap A g| \leqslant \frac{d \beta}{\varepsilon^{2}}(\varepsilon+d+2)|A|, \quad|A \cap A g| \geqslant\left(1-\frac{d \beta}{\varepsilon^{2}}(\varepsilon+d+2)\right)|A|
$$

holds, and one of the inequalities

$$
\left|A \cap A^{-1} g\right| \leqslant \frac{d \beta}{\varepsilon^{2}}(\varepsilon+d+2)|A|, \quad\left|A \cap A^{-1} g\right| \geqslant\left(1-\frac{d \beta}{\varepsilon^{2}}(\varepsilon+d+2)\right)|A|
$$

holds.

Define the subset $B_{+}$of $G$ by $B_{+}:=A \Delta(A g)^{c}$. The set $B_{+}^{c}$ is also equal to $\left(A \Delta(A g)^{c}\right)^{c}=A \Delta A g$. Note that

$$
\begin{aligned}
\left|B_{+}^{-1} S \Delta B_{+}\right| & \leqslant \sum_{s \in S}\left|B_{+}^{-1} s \Delta B_{+}\right| \\
& =\sum_{s \in S}\left|\left(\left(A \Delta(A g)^{c}\right)^{-1} s\right) \Delta\left(A \Delta(A g)^{c}\right)\right| \\
& =\sum_{s \in S}\left|\left(A^{-1} s \Delta\left((A g)^{c}\right)^{-1} s\right) \Delta\left(A \Delta(A g)^{c}\right)\right| \\
& =\sum_{s \in S}\left|\left(A^{-1} s \Delta\left((A g)^{c}\right)^{-1} s\right) \Delta\left(A^{c} \Delta A g\right)\right| \\
& =\sum_{s \in S}\left|\left(A^{-1} s \Delta A^{c}\right) \Delta\left(\left((A g)^{c}\right)^{-1} s \Delta A g\right)\right| \\
& =\sum_{s \in S}\left|\left(A^{-1} s \Delta A^{c}\right) \Delta\left(\left((A g)^{-1}\right)^{c} s \Delta A g\right)\right| \\
& =\sum_{s \in S}\left|\left(A^{-1} s \Delta A^{c}\right) \Delta\left((A g)^{-1} s \Delta(A g)^{c}\right)\right| \\
& \leqslant \sum_{s \in S}\left(\left|A^{-1} s \Delta A^{c}\right|+\left|(A g)^{-1} s \Delta(A g)^{c}\right|\right) \\
& \leqslant \frac{2 d \beta}{\varepsilon}(\varepsilon+d+2)|A|,
\end{aligned}
$$

and

$$
\begin{aligned}
\left|\left(B_{+}^{c}\right)^{-1} S \Delta B_{+}^{c}\right| & \leqslant \sum_{s \in S}\left|\left(B_{+}^{c}\right)^{-1} s \Delta B_{+}^{c}\right| \\
& =\sum_{s \in S}\left|\left((A \Delta A g)^{-1} s\right) \Delta(A \Delta A g)\right| \\
& =\sum_{s \in S}\left|\left(A^{-1} s \Delta(A g)^{-1} s\right) \Delta\left(A^{c} \Delta(A g)^{c}\right)\right| \\
& =\sum_{s \in S}\left|\left(A^{-1} s \Delta A^{c}\right) \Delta\left((A g)^{-1} s \Delta(A g)^{c}\right)\right| \\
& \leqslant \sum_{s \in S}\left(\left|A^{-1} s \Delta A^{c}\right|+\left|(A g)^{-1} s \Delta(A g)^{c}\right|\right) \\
& \leqslant \frac{2 d \beta}{\varepsilon}(\varepsilon+d+2)|A|
\end{aligned}
$$

hold as a consequence of Proposition 2.8(3). We consider the following cases, viz., $\left|B_{+}\right| \leqslant \frac{|G|}{2},\left|B_{+}\right|>\frac{|G|}{2}$. When $\left|B_{+}\right| \leqslant \frac{|G|}{2}$ holds, we obtain

$$
\varepsilon\left|B_{+}\right| \leqslant\left|B_{+}^{-1} S \backslash B_{+}\right| \leqslant\left|B_{+}^{-1} S \Delta B_{+}\right| \leqslant \frac{2 d \beta}{\varepsilon}(\varepsilon+d+2)|A|,
$$


which yields

Since

$$
\left|B_{+}\right| \leqslant \frac{2 d \beta}{\varepsilon^{2}}(\varepsilon+d+2)|A| .
$$

$|G|-\left|B_{+}\right|=\left|B_{+}^{c}\right|=|A \Delta A g|=|A|-|A \cap A g|+|A g|-|A \cap A g|=2|A|-2|A \cap A g|$

holds, we obtain

$$
2|A \cap A g| \leqslant|G|-2|A|+2|A \cap A g|=\left|B_{+}\right| \leqslant \frac{2 d \beta}{\varepsilon^{2}}(\varepsilon+d+2)|A| .
$$

While $\left|B_{+}\right|>\frac{|G|}{2}$ holds, we obtain

$$
\varepsilon\left|B_{+}^{c}\right| \leqslant\left|\left(B_{+}^{c}\right)^{-1} S \backslash B_{+}^{c}\right| \leqslant\left|\left(B_{+}^{c}\right)^{-1} S \Delta B_{+}^{c}\right| \leqslant \frac{2 d \beta}{\varepsilon}(\varepsilon+d+2)|A|,
$$

which yields

Since

$$
\left|B_{+}^{c}\right| \leqslant \frac{2 d \beta}{\varepsilon^{2}}(\varepsilon+d+2)|A| .
$$

$$
\left|B_{+}^{c}\right|=|A \Delta A g|=|A|-|A \cap A g|+|A g|-|A \cap A g|=2|A|-2|A \cap A g|
$$

holds, we obtain

$$
|A \cap A g| \geqslant|A|-\frac{d \beta}{\varepsilon^{2}}(\varepsilon+d+2)|A|=\left(1-\frac{d \beta}{\varepsilon^{2}}(\varepsilon+d+2)\right)|A| .
$$

Considering the subset $B_{-}$of $G$ defined by $B_{-}:=A \Delta\left(A^{-1} g\right)^{c}$, and using Proposition 2.8(5) and similar arguments as above, we obtain that

$$
\left|A \cap A^{-1} g\right| \leqslant \frac{d \beta}{\varepsilon^{2}}(\varepsilon+d+2)|A|
$$

or

$$
\left|A \cap A^{-1} g\right| \geqslant\left(1-\frac{d \beta}{\varepsilon^{2}}(\varepsilon+d+2)\right)|A|
$$

holds according as $\left|B_{-}\right| \leqslant \frac{|G|}{2}$ or $\left|B_{-}\right|>\frac{|G|}{2}$.

THEOREM 2.10. Let $C_{\Sigma}(G, S)$ be a non-bipartite $(n, d, \varepsilon)$-vertex expander for some $\varepsilon>0$. Then the eigenvalues of the normalised adjacency matrix of this graph are greater than $-1+\ell_{\varepsilon, d}$ with

$$
\ell_{\varepsilon, d}=\frac{\varepsilon^{4}}{2^{9} d^{8}} .
$$

Proof. On the contrary, let us assume that an eigenvalue of the normalised adjacency matrix of the graph $C_{\Sigma}(G, S)$ lies in the interval $\left[-1,-1+\ell_{\varepsilon, d}\right]$. Since $C_{\Sigma}(G, S)$ is nonbipartite, it follows that -1 is not an eigenvalue of its normalised adjacency matrix. Hence an eigenvalue of the normalised adjacency matrix of the graph $C_{\Sigma}(G, S)$ lies in the interval $\left(-1,-1+\ell_{\varepsilon, d}\right]$. Set

$$
\begin{aligned}
& \tau=d^{2} \sqrt{2 \ell_{\varepsilon, d}\left(2-\ell_{\varepsilon, d}\right)}, \\
& r=1-\frac{d \tau}{\varepsilon^{2}}(\varepsilon+d+2) .
\end{aligned}
$$

Since $\ell_{\varepsilon, d}=\frac{\varepsilon^{4}}{2^{9} d^{8}}$, we have

$$
\begin{gathered}
\tau=d^{2} \sqrt{2 \ell_{\varepsilon, d}\left(2-\ell_{\varepsilon, d}\right)}<d^{2} \sqrt{4 \ell_{\varepsilon, d}} \leqslant \frac{\varepsilon^{2}}{8 \sqrt{2} d^{2}}, \\
1-r=\frac{d \tau}{\varepsilon^{2}}(\varepsilon+d+2)<\frac{1}{8 \sqrt{2} d}(\varepsilon+d+2) \leqslant \frac{1}{8 \sqrt{2} d}(d-1+d+2) \leqslant \frac{3}{8 \sqrt{2}}<\frac{1}{3} .
\end{gathered}
$$


Consequently,

$$
\ell_{\varepsilon, d} \leqslant \frac{\varepsilon^{2}}{4 d^{4}}, \tau<\frac{\varepsilon^{2}}{4 d(d+1)} \text { and } r>\frac{2}{3} .
$$

Define the subsets $H_{+}, H_{-}$of $G$ by

$$
\begin{aligned}
& H_{+}:=\{g \in G:|A \cap A g| \geqslant r|A|\}, \\
& H_{-}:=\left\{g \in G:\left|A \cap A^{-1} g\right| \geqslant r|A|\right\} .
\end{aligned}
$$

Note that $H_{+}$contains the identity element of $G$. By the triangle inequality,

$$
\begin{aligned}
|A \backslash A g h| & \leqslant|A \backslash A h|+|A h \backslash A g h| \\
& =|A \backslash A h|+|A \backslash A g| \\
& =|A|-|A \cap A h|+|A|-|A \cap A g| \\
& \leqslant 2|A|-2 r|A| .
\end{aligned}
$$

Consequently,

$$
|A \cap A g h|=|A|-|A \backslash A g h| \geqslant|A|-2|A|+2 r|A|=(2 r-1)|A| .
$$

If $|A \cap A g h| \leqslant(1-r)|A|$, then we obtain

$$
(1-r)|A| \geqslant|A \cap A g h| \geqslant(2 r-1)|A|,
$$

which implies $r \leqslant \frac{2}{3}$. Since $r>\frac{2}{3}$, by Proposition 2.9(1), it follows that $H_{+}$contains $g h$. So $H_{+}$is a subgroup of $G$. Note that $H_{+}$is not equal to $G$, otherwise, we will obtain

$$
|A| \cdot \frac{|G|}{2} \geqslant|A|^{2}=\sum_{g \in G}|A \cap A g| \geqslant|G| \cdot r|A|,
$$

which yields $r \leqslant \frac{1}{2}$.

The following estimate

$$
|A|^{2}=\sum_{g \in G}|A \cap A g| \leqslant\left|H_{+}\right||A|+\frac{d \tau}{\varepsilon^{2}}(\varepsilon+d+2)|A|\left|G \backslash H_{+}\right|
$$

implies

$$
|A| \leqslant\left|H_{+}\right|+\frac{d \tau}{\varepsilon^{2}}(\varepsilon+d+2)\left(|G|-\left|H_{+}\right|\right) .
$$

Using Proposition 2.8(1), we obtain

$$
\frac{1}{2+\tau+\frac{d \tau}{\varepsilon}}|G|-\frac{d \tau}{\varepsilon^{2}}(\varepsilon+d+2)|G| \leqslant\left(1-\frac{d \tau}{\varepsilon^{2}}(\varepsilon+d+2)\right)\left|H_{+}\right| .
$$

We claim that $H_{+}$is a subgroup of $G$ of index two. To prove this claim, it suffices to show that

$$
\frac{1}{3}\left(1-\frac{d \tau}{\varepsilon^{2}}(\varepsilon+d+2)\right)<\frac{1}{2+\tau+\frac{d \tau}{\varepsilon}}-\frac{d \tau}{\varepsilon^{2}}(\varepsilon+d+2),
$$

i.e.

which is equivalent to

$$
\left(2+\tau+\frac{d \tau}{\varepsilon}\right)\left(1+\frac{2 d \tau}{\varepsilon^{2}}(\varepsilon+d+2)\right)<3,
$$

$$
\left(\tau+\frac{d \tau}{\varepsilon}\right)+\frac{2 d \tau}{\varepsilon^{2}}(\varepsilon+d+2)\left(2+\tau+\frac{d \tau}{\varepsilon}\right)<1 .
$$

Let $R=\tau+\frac{d \tau}{\varepsilon}$. From (3), we obtain

$$
\tau<\frac{1}{8 \sqrt{2}}\left(1-\frac{1}{d}\right)^{2}, \frac{d \tau}{\varepsilon}<\frac{1}{8 \sqrt{2}}\left(1-\frac{1}{d}\right) \text { and } R<\frac{1}{8 \sqrt{2}}\left(2-\frac{3}{d}+\frac{1}{d^{2}}\right) .
$$


From (8), it suffices to show that

$$
R+\frac{1}{4 \sqrt{2}}\left(2+\frac{1}{d}\right)(2+R)<1
$$

i.e. it suffices to show that

$$
\frac{1}{8 \sqrt{2}}\left(2-\frac{3}{d}+\frac{1}{d^{2}}\right)+\frac{1}{4 \sqrt{2}}\left(2+\frac{1}{d}\right)\left(2+\frac{1}{8 \sqrt{2}}\left(2-\frac{3}{d}+\frac{1}{d^{2}}\right)\right)<1 .
$$

Collecting the terms, it suffices to show that,

$$
\left(\frac{5}{4 \sqrt{2}}+\frac{1}{16}\right)+\left(\frac{1}{8 \sqrt{2}}-\frac{1}{16}\right) \frac{1}{d}+\left(\frac{1}{8 \sqrt{2}}-\frac{1}{64}\right) \frac{1}{d^{2}}+\frac{1}{64} \frac{1}{d^{3}}<1,
$$

which reduces to

$$
(60-40 \sqrt{2}) d^{3}-4(\sqrt{2}-1) d^{2}-(4 \sqrt{2}-1) d-1>0 .
$$

The above cubic polynomial in $d$ is positive for $d \geqslant 2$ and hence the claim that $H_{+}$is a subgroup of $G$ of index two follows.

By Proposition 2.8(2), $H_{-}$does not intersect the set $S$. Similar to as before, the following estimate

$$
|A|^{2}=\sum_{g \in G}\left|A \cap A^{-1} g\right| \leqslant\left|H_{-}\right||A|+\frac{d \tau}{\varepsilon^{2}}(\varepsilon+d+2)|A|\left|G \backslash H_{-}\right|
$$

implies

$$
|A| \leqslant\left|H_{-}\right|+\frac{d \tau}{\varepsilon^{2}}(\varepsilon+d+2)\left(|G|-\left|H_{-}\right|\right) .
$$

This inequality combined with Proposition 2.8(1) yields

$$
\frac{1}{2+\tau+\frac{d \tau}{\varepsilon}}|G|-\frac{d \tau}{\varepsilon^{2}}(\varepsilon+d+2)|G| \leqslant\left(1-\frac{d \tau}{\varepsilon^{2}}(\varepsilon+d+2)\right)\left|H_{-}\right| .
$$

The inequality in (7) (which has been established) implies that

$$
\left|H_{-}\right|>\frac{|G|}{3}
$$

and consequently, $H_{-}$is nonempty. Note that for $h_{-} \in H_{-}, h_{+} \in H_{+}$, the triangle inequality implies

$$
\begin{aligned}
\left|A \backslash A^{-1} h_{-} h_{+}\right| & \leqslant\left|A \backslash A h_{+}\right|+\left|A h_{+} \backslash A^{-1} h_{-} h_{+}\right| \\
& =\left|A \backslash A h_{+}\right|+\left|A \backslash A^{-1} h_{-}\right| \\
& =|A|-\left|A \cap A h_{+}\right|+|A|-\left|A \cap A^{-1} h_{-}\right| \\
& \leqslant 2|A|-2 r|A|,
\end{aligned}
$$

which yields

$$
\left|A \cap A^{-1} h_{-} h_{+}\right|=|A|-\left|A \backslash A^{-1} h_{-} h_{+}\right| \geqslant|A|-2|A|+2 r|A|=(2 r-1)|A| .
$$

If $\left|A \cap A^{-1} h_{-} h_{+}\right| \leqslant(1-r)|A|$, then we will obtain

$$
(1-r)|A| \geqslant\left|A \cap A^{-1} h_{-} h_{+}\right| \geqslant(2 r-1)|A|,
$$

which in turn implies $r \leqslant \frac{2}{3}$. Since $r>\frac{2}{3}$, using Proposition 2.9(2), we conclude that $\left|A \cap A^{-1} h_{-} h_{+}\right| \geqslant r|A|$, i.e. $H_{-}$contains $h_{-} h_{+}$. Thus, $H_{-} H_{+}$is contained in $H_{-}$. Since $H_{-}$is a nonempty proper subset of $G$, it follows that $H_{-}$is equal to $H_{+}$or $H_{-}$is equal to the non-trivial coset of $H_{+}$in $G$, i.e. $G \backslash H_{+}$. Note that if $H_{-}$is not equal to $H_{+}$, then the index two subgroup $H_{+}$of $G$ will contain $S$ (since $H_{-} \cap S=\varnothing$ ). Since the graph $C_{\Sigma}(G, S)$ is an expander, it is connected (otherwise, for a connected component $C$ of least size, the ratio of the size of the boundary of $C$ to the size of $C$ would be 
zero, which is impossible in this case). Thus, every element of $G$ is connected to the identity element. So, any element of $G$ can be expressed as a product of elements of the set $S \cup S^{-1}$. This shows that $G$ is contained in $H_{+}$, which is impossible. So $H_{-}$ is equal to $H_{+}$. Consequently, $H_{+}$is a subgroup of $G$ of index two avoiding $S$. Thus, the graph $C_{\Sigma}(G, S)$ is bipartite by Lemma 2.5. We are done.

Proof of Theorem 1.4. Since $C_{\Sigma}(G, S)$ is connected, its vertex Cheeger constant $h_{\Sigma}(G)$ is positive. Thus $C_{\Sigma}(G, S)$ is an $h_{\Sigma}(G)$-expander with $h_{\Sigma}(G)>0$. So Theorem 1.4 follows from Theorem 2.10.

Proof of Corollary 1.6. From Theorem 1.4, it follows that for any $k \geqslant 1$, the eigenvalues of the normalised adjacency matrix of $C_{\Sigma}\left(G_{k}, S_{k}\right)$ are greater than $-1+\frac{\varepsilon^{4}}{2^{9} d^{8}}$, which depends on $\varepsilon, d$, but not on $k$. Hence the corollary.

In the context of Cayley graphs, the first author obtained the following bound on the spectrum of the adjacency operator.

TheOREM 2.11 ([3, Theorem 1.4]). Let $\mathcal{S}$ be a symmetric subset of $G$ and let $C(G, \mathcal{S})$ denote the Cayley graph of $G$ with respect to $\mathcal{S}$ with $|\mathcal{S}|=d$ and $h(G)$ denote its vertex Cheeger constant. If $C(G, \mathcal{S})$ is non-bipartite, then the largest eigenvalue of its normalised Laplacian matrix is less than

$$
\lambda_{n}<2-\frac{h(G)^{4}}{2^{9} d^{6}(d+1)^{2}} .
$$

As a consequence of the proof of Theorem 2.10, we obtain the following refinement of the bound provided in the above result.

THEOREM 2.12. Let $\mathcal{S}$ be a symmetric subset of $G$ and let $C(G, \mathcal{S})$ denote the Cayley graph of $G$ with respect to $\mathcal{S}$ with $|\mathcal{S}|=d$ and $h(G)$ denote its vertex Cheeger constant. Suppose $\mathcal{S}$ does not contain the identity element. If the graph $C(G, \mathcal{S})$ is non-bipartite, then the largest eigenvalue of its normalised Laplacian matrix is less than

$$
2-\frac{h(G)^{4}}{2^{9} d^{8}}
$$

Proof. Suppose $C(G, \mathcal{S})$ is an $\epsilon$-vertex expander with $\epsilon>0$ and it is non-bipartite. We claim that the largest eigenvalue of the normalised Laplacian matrix is less than

$$
2-\frac{\epsilon^{4}}{2^{9} d^{8}} \text {. }
$$

The bound on this eigenvalue given by [3, Theorem 1.4] is

$$
2-\frac{\epsilon^{4}}{2^{9} d^{6}(d+1)^{2}} .
$$

Note that the proof of this result as in loc.cit. crucially relies on the last inequality in $[3$, p. 306], i.e. the inequality

$$
\left(\beta+\frac{d \beta}{\epsilon}\right)+\frac{2 d \beta}{\epsilon^{2}}(\epsilon+d+2)\left(2+\beta+\frac{d \beta}{\epsilon}\right)<1
$$

where $\beta=d^{2} \sqrt{2 \zeta(2-\zeta)}$. This inequality has been established using $\epsilon \leqslant d$ and the hypothesis that $\zeta=\frac{\epsilon^{4}}{2^{9} d^{6}(d+1)^{2}}$. The analogue of $(9)$ in the context of Cayley sum graph is the inequality

$$
\left(\tau+\frac{d \tau}{\varepsilon}\right)+\frac{2 d \tau}{\varepsilon^{2}}(\varepsilon+d+2)\left(2+\tau+\frac{d \tau}{\varepsilon}\right)<1
$$


in (8) where $\tau=d^{2} \sqrt{2 \ell_{\varepsilon, d}\left(2-\ell_{\varepsilon, d}\right)}$. The above inequality has been established using $\varepsilon \leqslant d-1$ and $\ell_{\varepsilon, d}=\frac{\varepsilon^{4}}{2^{9} d^{8}}$. Hence (9) will be satisfied for $\zeta=\frac{\varepsilon^{4}}{2^{9} d^{8}}$ if $\epsilon \leqslant d-1$ holds, which follows from Proposition 2.7. This shows the claim. Noting that $C(G, \mathcal{S})$ is an $h(G)$-vertex expander, and $h(G)>0$ (since the graph $C(G, \mathcal{S})$ is connected), the result follows from the claim.

Acknowledgements. We thank the anonymous reviewers for a careful reading of the article and for their valuable comments and suggestions that improved the work. We wish to thank Emmanuel Breuillard for a number of helpful discussions during the opening colloquium of the Münster Mathematics Cluster. The first author would like to acknowledge the support of the OWLF program of the Mathematisches Forschungsinstitut Oberwolfach (MFO). The second author would like to acknowledge the Initiation Grant from the Indian Institute of Science Education and Research Bhopal and the INSPIRE Faculty Award from the Department of Science and Technology, Government of India. He would also like to thank the MFO for their hospitality.

\section{REFERENCES}

[1] Noga Alon, Eigenvalues and expanders, Combinatorica 6 (1986), no. 2, 83-96, Theory of computing (Singer Island, Fla., 1984).

[2] Noga Alon and Vitali D. Milman, $\lambda_{1}$, isoperimetric inequalities for graphs, and superconcentrators, J. Combin. Theory Ser. B 38 (1985), no. 1, 73-88.

[3] Arindam Biswas, On a Cheeger type inequality in Cayley graphs of finite groups, European J. Combin. 81 (2019), 298-308.

[4] Emmanuel Breuillard, Ben Green, Robert Guralnick, and Terence Tao, Expansion in finite simple groups of Lie type, J. Eur. Math. Soc. (JEMS) 17 (2015), no. 6, 1367-1434.

[5] Peter Buser, A note on the isoperimetric constant, Ann. Sci. École Norm. Sup. (4) 15 (1982), no. $2,213-230$.

[6] Jeff Cheeger, A lower bound for the smallest eigenvalue of the Laplacian, in Problems in analysis (Papers dedicated to Salomon Bochner, 1969), Princeton University Press, 1970, pp. 195-199.

[7] Fan R. K. Chung, Diameters and eigenvalues, J. Amer. Math. Soc. 2 (1989), no. 2, 187-196.

[8] _ Laplacians of graphs and Cheeger's inequalities, in Combinatorics, Paul Erdös is eighty, Vol. 2 (Keszthely, 1993), Bolyai Soc. Math. Stud., vol. 2, János Bolyai Math. Soc., Budapest, 1996, pp. 157-172.

[9] Matt DeVos, Luis Goddyn, Bojan Mohar, and Robert Šámal, Cayley sum graphs and eigenvalues of (3,6)-fullerenes, J. Combin. Theory Ser. B 99 (2009), no. 2, 358-369.

[10] Gregory A. Freimman, Groups and the inverse problems of additive number theory, in Numbertheoretic studies in the Markov spectrum and in the structural theory of set addition (Russian), Kalinin Gos. Univ., 1973, pp. 175-183.

[11] Ben Green, Counting sets with small sumset, and the clique number of random Cayley graphs, Combinatorica 25 (2005), no. 3, 307-326.

[12] (2017), no. 2, 248-266.

[13] Ben Green and Robert Morris, Counting sets with small sumset and applications, Combinatorica 36 (2016), no. 2, 129-159.

[14] David Grynkiewicz, Vsevolod F. Lev, and Oriol Serra, Connectivity of addition Cayley graphs, J. Combin. Theory Ser. B 99 (2009), no. 1, 202-217.

[15] Sergeř V. Konyagin and Il'ya D. Shkredov, On subgraphs of random Cayley sum graphs, European J. Combin. 70 (2018), 61-74.

[16] Alexander Lubotzky, Discrete groups, expanding graphs and invariant measures, Progress in Mathematics, vol. 125, Birkhäuser Verlag, Basel, 1994, With an appendix by Jonathan D. Rogawski.

[17] Xuanlong Ma, Min Feng, and Kaishun Wang, Subgroup perfect codes in Cayley sum graphs, Des. Codes Cryptogr. 88 (2020), no. 7, 1447-1461.

[18] Rudi Mrazović, Extractors in Paley graphs: a random model, European J. Combin. 54 (2016), $154-162$. 
Arindam Biswas, Department of Mathematics, Technion - Israel Institute of Technology, Haifa 32000 , Israel

E-mail : biswas@campus.technion.ac.il; arin.math@gmail.com

Jyoti Prakash SAha, Department of Mathematics, Indian Institute of Science Education and Research Bhopal, Bhopal Bypass Road, Bhauri, Bhopal 462066, Madhya Pradesh, India E-mail : jpsaha@iiserb.ac.in 